\title{
The role of symmetry in the short-time critical dymamics
}

\author{
Tânia Tomé \\ Instituto de Física \\ Universidade de São Paulo \\ Caixa Postal 66318 \\ 05315-970 São Paulo, São Paulo, Brazil
}

(Dated: November 2, 2018)

\begin{abstract}
We show that the short-time critical exponent $\theta$ related to the critical initial slip in a stochastic model can be determined by the time correlation of the order parameter. In our procedure it suffices to start with an uncorrelated state with zero order parameter instead of departing, as usually done, from an initial state with a nonzero order parameter. The proof uses the group of symmetry operations related to the Markovian dynamics. Our scheme is extended to cover models with absorbing states.
\end{abstract}

PACS numbers: 05.70.Ln, 05.50.+q, 64.60.Ht 


\section{INTRODUCTION}

The universal behavior occurring in the first steps of a Monte Carlo simulation, the shorttime dynamics, has been amply investigated [1, 2, 3, 4, 5, 6, 6] in the last years. According to renormalization group arguments [1] the early time behavior of the order parameter (the magnetization, for example, in the case of the Ising model) follows a power law with a critical universal exponent $\theta$. The numerical calculation of the exponent $\theta$ is performed by placing the system at the critical point and departing from a configuration where the order parameter $m_{0}$ is very small.

The purpose of this article is to show that it is possible to determine the exponent $\theta$ by starting with a configuration in which the order parameter is identically zero. In our approach we do not measure the order parameter itself, which is zero, but its time coorelation. If $M(t)$ denotes de instantaneous order parameter we show that the quantity

$$
Q(t)=\langle M(t) M(0)\rangle
$$

follows a power law

$$
Q(t) \sim t^{\theta}
$$

where the initial configuration is uncorrelated and such that

$$
\langle M(0)\rangle=0
$$

This result is general and can be applied to any lattice system described by a Markovian process and such that the transition probability is invariant under a given group of symmetry operations. In this procedure, which was previously deduced for models with up-down symmetry [8]. Here we generalize the scheme to inclued models with other types of symmetries. In particular we apply the method to systems with antiferromagnetic ordering and to the Baxter-Wu model in a triangular lattice [9]. Employing this procedure it was recently calculated by numerical simulations [10], with an excellent precision, the exponent $\theta$ associated to the Baxter-Wu model with triplet interactions in a triangular lattice.

We consider also the short-time behavior of intrinsic irreversible models with absorbing states, such as the contact process. In this case, we demonstrate here that it suffices to depart from a configuration with just one occupied site instead of starting from a configuration

where the density of particles is finite and small. Therefore, the short-time behavior of the 
order parameter is identical to the behavior found by time-dependent simulations [11, 12] departing from a unique initial seed. In both methods it is necessary to place the system in its critical point.

\section{TRANSITION PROBABILITY AND SYMMETRY}

In this section and in the following we develop the formalism leading to the expression (11). For this purpose we consider the class of Markovian processes defined on a lattice whose probability distribution $P(\sigma, t)$ satisfies the equation

$$
P(\sigma, t)=\sum_{\sigma^{\prime}} T\left(\sigma, \sigma^{\prime}, t\right) P_{0}\left(\sigma^{\prime}\right)
$$

where $T\left(\sigma, \sigma^{\prime}, t\right)$ is the probability of reaching the configuration $\sigma$ from configuration $\sigma^{\prime}$ in an interval of time $t$ and $P_{0}\left(\sigma^{\prime}\right)$ is the initial probability distribution. We use the notation $\sigma=\left(\sigma_{1}, \sigma_{2}, \ldots, \sigma_{N}\right)$ where $N$ is the number of sites in the lattice and $\sigma_{i}$ is the random variable attached to the $i$-th site and that takes two values.

If the system evolves in time according to a master equation (continuous time Markovian process)

$$
\frac{d}{d t} P(\sigma, t)=\sum_{\sigma^{\prime}}\left\{W\left(\sigma, \sigma^{\prime}\right) P\left(\sigma^{\prime}, t\right)-W\left(\sigma^{\prime}, \sigma\right) P(\sigma, t)\right\}
$$

then the transition probability $T\left(\sigma, \sigma^{\prime}, t\right)$ are the elements of the matrix $T$ given by

$$
\widehat{T}=\exp \{t \widehat{W}\}
$$

where $\widehat{W}$ is the matrix whose elements are

$$
\widehat{W}\left(\sigma^{\prime}, \sigma\right)=W\left(\sigma^{\prime}, \sigma\right) \quad \sigma^{\prime} \neq \sigma
$$

and

$$
\widehat{W}(\sigma, \sigma)=-\sum_{\sigma^{\prime}(\neq \sigma)} W\left(\sigma^{\prime}, \sigma\right)
$$

Let $R$ be a symmetry operation that leaves the transition probability invariant, or, equivalently, the matrix $W$ invariant, that is

$$
W\left(R \sigma, R \sigma^{\prime}\right)=W\left(\sigma, \sigma^{\prime}\right)
$$

and by consequence

$$
T\left(R \sigma, R \sigma^{\prime}, t\right)=T\left(\sigma, \sigma^{\prime}, t\right)
$$


For simplicity, we will consider here only models in which the symmetry operation $R$ changes the sign of the order parameter, that is,

$$
M(R \sigma)=-M(\sigma)
$$

and that $M(\sigma)$ is linear in $\sigma$, that is,

$$
M(\sigma)=\sum_{i} \mu_{i} \sigma_{i}
$$

\section{TIME-DEPENDENT BEHAVIOR}

We will focus on the time-dependent behavior of the average

$$
\langle M(\sigma)\rangle_{t}=\sum_{\sigma} M(\sigma) P(\sigma, t)
$$

of the order parameter $M(\sigma)$. Its time evolution is given by

$$
\langle M(\sigma)\rangle_{t}=\sum_{\sigma} \sum_{\sigma^{\prime}} M(\sigma) T\left(\sigma, \sigma^{\prime}, t\right) P_{0}\left(\sigma^{\prime}\right)
$$

where the initial state $P_{0}(\sigma)$ is uncorrelated with a nonzero magnetization. That is, the initial magnetization

$$
\langle M(\sigma)\rangle_{0}=\sum_{\sigma} M(\sigma) P_{0}(\sigma)=N m_{0}
$$

is nonzero, where $N$ is the number of sites of the lattice and $m_{0}$ is a small quantity. As stated by the short-time scaling theory, the order parameter folows, at the critical point, a power law behavior

$$
\langle M(\sigma)\rangle_{t} \sim m_{0} t^{\theta}
$$

for small values of $m_{0}$. According to this theory yet the initial state is prepared in such a way that all sites are uncorrelated with a nonzero (and small) initial order parameter $m_{0}$. In order to set up such an initial state, one attributes to each site a magnetization $m_{i}=m_{0} \mu_{i}$. Or equivalentely, the spin of the $i$-th site will be $\sigma_{i}=\mu_{i}$ with probability $\left(1+m_{0}\right) / 2$ and will be $\sigma_{i}=-\mu$ with probability $\left(1-m_{0}\right) / 2$. The initial probability $P_{0}(\sigma)$ can then be written as

$$
P_{0}(\sigma)=\Phi_{0} \prod_{i}\left\{1+m_{0} \mu_{i} \sigma_{i}\right\}
$$

where

$$
\Phi_{0}=\frac{1}{2^{N}}
$$


Notice that using equation (12) and (17) we can trivially find that

$$
\langle M(\sigma)\rangle_{0}=\sum_{\sigma} M(\sigma) P_{0}(\sigma)=\operatorname{Nam}_{0}
$$

where $a$ is the constant

$$
a=\frac{1}{N} \sum_{j}\left[\mu_{j}\right]^{2}
$$

For small values of $m_{0}$, the expansion of the initial probability $P_{0}(\sigma)$ in powers of $m_{0}$ gives, up to linear terms in $m_{0}$ the following expression

$$
P_{0}(\sigma)=\Phi_{0}\left\{1+m_{0} M(\sigma)\right\}
$$

Substituting this expression in equation (14) we get

$$
\begin{aligned}
& \langle M(\sigma)\rangle_{t}=\sum_{\sigma} \sum_{\sigma^{\prime}} M(\sigma) T\left(\sigma, \sigma^{\prime}, t\right) \Phi_{0}+ \\
& +\sum_{\sigma} \sum_{\sigma^{\prime}} M(\sigma) T\left(\sigma, \sigma^{\prime}, t\right) \Phi_{0} m_{0} M\left(\sigma^{\prime}\right)
\end{aligned}
$$

Now, the first term on the right hand side vanishes identically due to the following reasoning. Since the symmetry operation $R$ leaves the transition probability invariant but changes the sign of the order parameter, we have

$$
\sum_{\sigma} \sum_{\sigma^{\prime}} M(R \sigma) T\left(R \sigma, R \sigma^{\prime}, t\right) \Phi_{0}=-\sum_{\sigma} \sum_{\sigma^{\prime}} M(\sigma) T\left(\sigma, \sigma^{\prime}, t\right) \Phi_{0}
$$

By a change of variable, $R \sigma \rightarrow \sigma$, the left-hand side of this equation equals the first term of the right-hand side of equation (22) so that it vanishes. Therefore

$$
Q(t)=\lim _{m_{0} \rightarrow 0} \frac{\langle M(\sigma)\rangle_{t}}{m_{0}}=\sum_{\sigma} \sum_{\sigma^{\prime}} M(\sigma) T\left(\sigma, \sigma^{\prime}, t\right) M\left(\sigma^{\prime}\right) \Phi_{0}
$$

and, from equation (16) it follows that

$$
Q(t) \sim t^{\theta}
$$

\section{APPLICATIONS}

\section{A. Models with up-down symmetry}

We begin with a simple example, namely the ferromagnetic Ising model coupled to a stochastic dynamics such as the Metropolis algorithm. The order parameter is defined by

$$
M(\sigma)=\sum_{i} \sigma_{i}
$$


where the summation is over all sites of the lattice. For the present case $\mu_{i}=+1$ for all sites of the lattice. Here the symmetry operation $R$, with the properties given by equations (10) and (11), is the one in which the up-down symmetry is observed, that is, the operation that changes the sign of each spin variable $\sigma_{i} \rightarrow-\sigma_{i}$.

The short-time behavior for the Ising model has been already studied through the present formalism [8]. Besides, using this formalism, it has been possible to determine the short-time behavior for several irreversible models (i. e., lacking detailed balance) [8, 13, 14] with updown symmetry dynamics. These include for instance the majority vote model and similar nonequilibrium models [8, 15].

\section{B. Models with antiferromagnet ordering}

In this case the system is divided into two sublattices $A$ and $B$. The order parameter is defined by

$$
M(\sigma)=\sum_{i \in A} \sigma_{i}-\sum_{i \in B} \sigma_{i}
$$

Therefore, for this case one has $\mu_{i}=+1$ if $i \in A$ and $\mu_{i}=-1$ if $i \in B$. The symmetry operation $R$ is a translation such that a given site of one sublattice goes into a site of the other sublattice.

\section{Baxter-Wu model}

We consider in this subsection the Baxter-Wu model with triplet interactions defined on a triangular lattice [9, 10]. The lattice is composed of three sublattices which we denote by $A, B$, and $C$. The Baxter-Wu model does not have a global symmetry but semi-global symmetries. The Hamiltonian of the model and a fortiori the transition probability is invariant if we change the signs of two sublattices leaving the third invariant. It is convenient therefore to define three symmetry operations, denoted by $R_{A}, R_{B}$ and $R_{C}$. The symmetry operation $R_{A}$ changes the signs of the spins belonging to the sublattices $B$ and $C$ and leaves the signs of the spins of sublattice $A$ invariant. Similar definitions can be stated for $R_{B}$ and $R_{C}$. Each of these symmetry operations leaves the Baxter-Wu Hamiltonian invariant and a fortiori the transition probability invariant. 
We take as the order parameter the magnetization of one of the sublattices, say, sublattice $A$, given by

$$
M_{A}(\sigma)=\sum_{i \in A} \sigma_{i}
$$

Comparing it with equation (12) we have that $\mu_{i}=1$ if $i \in A$ and $\mu_{i}=0$ if $i \in B$ or $i \in C$. The symmetry operation $R_{B}$ (or $R_{C}$ ) changes the sign of $M_{A}(\sigma)$ and leaves the transition probability invariant. According to the formalism developed in the previous section we conclude that

$$
Q_{A}(t)=\lim _{m_{0} \rightarrow 0} \frac{\left\langle M_{A}(\sigma)\right\rangle_{t}}{m_{0}}=\sum_{\sigma} \sum_{\sigma^{\prime}} M_{A}(\sigma) T\left(\sigma, \sigma^{\prime}, t\right) M_{A}\left(\sigma^{\prime}\right) \Phi_{0}
$$

will behave as

$$
Q_{A}(t) \sim t^{\theta}
$$

Equivalently, we may demonstrate that the analogous quantities $Q_{B}(t)$ and $Q_{C}(t)$ related to the magnetizations $M_{B}(\sigma)$ and $M_{C}(\sigma)$ of sublattices $B$ and $R_{C}$, respectively, will behave as $t^{\theta}$.

We may also use as the order parameter the total magnetization

$$
M(\sigma)=\sum_{i} \sigma_{i}
$$

which we write as the sum of the magnetizations of the three sublattices

$$
M(\sigma)=M_{A}(\sigma)+M_{B}(\sigma)+M_{C}(\sigma)
$$

which leads to

$$
Q(t)=\sum_{\sigma} \sum_{\sigma^{\prime}} M(\sigma) T\left(\sigma, \sigma^{\prime}, t\right) M\left(\sigma^{\prime}\right) \Phi_{0}
$$

Substituting (32) into (33) we see that $Q(t)$ is a sum of nine terms. The terms that involve magnetizations of distinct sublattices will vanish. For instance, the term that involve $M_{A}$ and $M_{B}$ will change sign by the use o the symetry operation $R_{A}$. The nonvanishing terms are the three terms that involve the same magnetization. One concludes that the quantity

$$
Q(t)=Q_{A}(t)+Q_{B}(t)+Q_{C}(t)
$$

and therefore will behave as $t^{\theta}$. This procedure was used [10] to determine the exponent $\theta$. The numerical results give very precise values for the exponent when compared with the results coming from simulations with nonzero initial magnetization. 


\section{CONTACT MODEL}

Now we discuss the short-time behavior fo models with an absorbing state. These models do not possess symmetry operations like the ones defined in the preceeding sections. Due to this fundamental difference we need to proceed by introducing another approach. The simplest example of this type of model is the contact process [12]. Such model is defined in a lattice and each microscopic state is identified with $\sigma=\left(\sigma_{1}, \sigma_{2}, \ldots, \sigma_{N}\right)$ where $\sigma_{i}=0$ or 1 according wether the site $i$ is empty or occupied by a particle. It evolves in time according to local Markovian rules where particles are catalitically created and spontaneously anihilated.

The initial probability is such that all sites are uncorrelated and such that the average $\left\langle\sigma_{i}\right\rangle=\rho_{0}$, that is,

$$
P_{0}(\sigma)=\prod_{i}\left\{a\left(1-\sigma_{i}\right)+b \sigma_{i}\right\}
$$

where

$$
a=1-\rho_{0} \quad b=\rho_{0}
$$

is the total number of sites in the lattice.

Following the short-time scaling theory the order parameter $\langle n(\sigma)\rangle$ given by

$$
\langle n(\sigma)\rangle_{t}=\sum_{\sigma^{\prime}} \sum_{\sigma} n(\sigma) T\left(\sigma, \sigma^{\prime}, t\right) P_{0}\left(\sigma^{\prime}\right)
$$

where

$$
n(\sigma)=\sum_{i} \sigma_{i}
$$

is the number of particle, behaves, at the critical point, as

$$
\langle n(\sigma)\rangle \sim \rho_{0} t^{\theta}
$$

Consequently the quantity

$$
Q(t)=\frac{1}{N} \lim _{\rho_{0} \rightarrow 0} \frac{\langle n(\sigma)\rangle_{t}}{\rho_{0}}
$$

has a similar behavior in the early time regime

$$
Q(t) \sim t^{\theta}
$$

For small values $\rho_{0}$ of we have

$$
P_{0}(\sigma)=a^{N} \Phi_{0}(\sigma)+a^{N-1} b \sum_{j} \Phi_{j}(\sigma)
$$


where

$$
\Phi_{0}(\sigma)=\prod_{i}\left(1-\sigma_{i}\right)
$$

is the probability distribution such that the configuration $(0,0,0, \ldots, 0)$ (all sites empty) has probability 1 and the other configurations have zero probability,

$$
\Phi_{j}(\sigma)=\sigma_{j} \prod_{i(\neq j)}\left(1-\sigma_{i}\right)
$$

is the probability distribution such that the configuration $(0,0, \ldots, 1, \ldots 0)$ (a particle placed at the $j$-th site and all other sites empty) has probability 1 and all other configurations have zero probability.

The average $\langle n(\sigma)\rangle_{t}$ can then be written as a sum of two parts

$$
\begin{gathered}
\langle n(\sigma)\rangle_{t}=a^{N} \sum_{\sigma^{\prime}} \sum_{\sigma} n(\sigma) T\left(\sigma, \sigma^{\prime}, t\right) \Phi_{0}\left(\sigma^{\prime}\right)+ \\
+a^{N-1} b \sum_{j} \sum_{\sigma^{\prime}} \sum_{\sigma} n(\sigma) T\left(\sigma, \sigma^{\prime}, t\right) \Phi_{j}\left(\sigma^{\prime}\right)
\end{gathered}
$$

Since the contact process has an abosrbing state devoided of particles, the first term vanishes identically because $\Phi_{0}(\sigma)$ is the absorbing state. Therefore, using the translational invariance we obtain

$$
Q(t)=\sum_{\sigma^{\prime}} \sum_{\sigma} n(\sigma) T\left(\sigma, \sigma^{\prime}, t\right) \Phi_{j}\left(\sigma^{\prime}\right)
$$

To calculate numerically $Q(t)$, we start from a configuration with just one occupied site and determine the number of occupied sites at time $t$.

According to the scaling relations established for the time-dependent behavior of the contact model in which the simulation is started with just one occupied site, the average number of particles $n_{p}(t)$ behaves as [11, 12]

$$
n_{p}(t) \sim t^{\eta}
$$

As $Q(t)$ is identified with $n_{p}(t)$ so the exponent $\theta$ is identified with the exponent $\eta$ [16].

Let us now calculate the time correlation of a given site, say the site $j$. It is given by

$$
A(t)=\sum_{\sigma^{\prime}} \sum_{\sigma} \sigma_{j} T\left(\sigma, \sigma^{\prime}, t\right) \sigma_{j}^{\prime} P_{0}\left(\sigma^{\prime}\right)
$$

and behaves, according to the short-time scaling theory, as

$$
A(t) \sim t^{\lambda}
$$


Now

$$
\sigma_{j} P_{0}(\sigma)=b \sigma_{j} \prod_{i(\neq j)}\left\{a\left(1-\sigma_{i}\right)+b \sigma_{i}\right\}
$$

where we have used the obvious relations $\sigma_{j}\left(1-\sigma_{j}\right)=0$ and $\sigma_{j} \sigma_{j}=\sigma_{j}$. Therefore, in the limit $\rho_{0} \rightarrow 0$, we get

$$
\lim _{\rho_{0} \rightarrow 0} \frac{1}{\rho_{0}} \sigma_{j} P_{0}(\sigma)=\Phi_{j}(\sigma)
$$

Consequently,

$$
B(t)=\lim _{\rho_{0} \rightarrow 0} \frac{A(t)}{\rho_{0}}=\sum_{\sigma^{\prime}} \sum_{\sigma} \sigma_{j} T\left(\sigma, \sigma^{\prime}, t\right) \Phi_{j}\left(\sigma^{\prime}\right)
$$

so that $B(t)$ behaves as

$$
B(t) \sim t^{\lambda}
$$

Given that the initial particle seed is placed at a given site, the quantity $B(t)$ is the probability that this site be occupied at time $t$.

The exponent $\lambda$ is related to dynamic exponent $z$ by $\lambda=d / z-\theta$ [8]. Since the exponent $\theta$ was identified as the exponent $\eta$, it follows that $\lambda=d / z-\eta$. Now, from the hyperscaling

relation for the contact process we have $d / z-\eta=2 \delta$ [12] where $\delta$ is the exponent associated to the survival probability. Therefore it follows that $\lambda=2 \delta$.

¿From the formalism developed here we conclude that the study of the short-time behavior of the contact process, discussed in reference [16], is as a matter of fact equivalent to the study of this model by means of the time-dependent technique. Moreover, the critical exponents associated to the short time dynamics for the contact model, as well as the relation among them, are equivalent to those found for the time-dependent simulations.

\section{CONCLUSION}

We have shown that the short-time critical exponent $\theta$ of several models invariant under a given group of symmetry can be calculated from the time correlation of the order parameter

$$
Q(t)=\langle M(t) M(0)\rangle
$$

where $\langle f(t) g(0)\rangle$ is a notation defined by

$$
\langle f(t) g(0)\rangle=\sum_{\sigma} \sum_{\sigma^{\prime}} f(\sigma) T\left(\sigma, \sigma^{\prime}, t\right) g\left(\sigma^{\prime}\right) \Phi_{0}
$$


We have also obtained similar formula for the contact process and shown that the shorttime critical exponent $\theta$ is equal to time-dependent critical exponent $\eta$. Finally, the results

obtained here for the continuous time Markovian processes can be straitforwardly extended to the probabilistic cellular automata (discrete time Markovian process).

\section{Acknowledgements}

I am very grateful to J. R. Drugowich de Felício for useful and instigating discussions about this issue. This work was suported in part by the Brazilian agency CNPq.

[1] H. K. Janssen, B. Schaub, and Schmittmann, Z. Phys. B 73, 539 (1989).

[2] D. A. Huse, Phys. Rev. B 40, 304 (1989).

[3] Z. B. Li, L. Schülke and B. Zheng, Phys. Rev. Lett. 74 ,3396 (1994).

[4] Z. B. Li, U. Ritschel, and B. Zheng, J. Phys. A 27, L837 (1994).

[5] B. Zheng, Int. J. Mod. Phys. B 12, 1419 (1998).

[6] B. Zheng and H. J. Luo, Phys. Rev. E 63, 066130 (2001).

[7] A. Jaster, J. Mainville, L. Shülke and B. Zheng, J. Phys. A 32, 1395 (1999).

[8] T. Tomé and M. J. de Oliveira, Phys. Rev. E 58, 4242 (1998).

[9] R. J. Baxter and F. Y. Wu, Phys. Rev. Lett. 31, 1294 (1973).

[10] E. Arashiro and J. R. D Drugowich de Felício. Submitted to publication (cond-mat/0212116).

[11] I. Jensen and R. Dickman, J. Stat. Phys. 71, 89 (1993).

[12] J. Marro and R. Dickman, Nonequilibrium Phase Transitions in Lattice Models, Cambridge University Press, Cambridge, 1999.

[13] T. Tomé and J. R. Drugowich de Felício, Mod. Phys. Lett. B 12, 873 (1998).

[14] T. Tomé, Braz. J. Phys. 30, 152 (2000).

[15] J. F. F. Mendes and M. A. Santos, Phys. Rev. E 57, 108 (1998).

[16] H. Hinrichsen, Advances in Physics 49, 815 (2000). 